\title{
Orbital mucormycosis with retinal and ciliary artery occlusions
}

\author{
LUO QINGLI,' JAMES C ORCUTT,' AND LEONARD S SEIFTER²
}

From the 'Department of Ophthalmology, University of Washington School of Medicine, Seattle, Washington, and the ${ }^{2}$ Eye Institute of Olympia, Olympia, Washington, USA

SUMMARY A 61-year-old man presented with acute, painful loss of vision in the left eye due to a central retinal artery occlusion. Fluorescein angiography confirmed the central retinal artery occlusion and also identified a nasal posterior ciliary artery occlusion. CT scanning revealed a left medial orbital mass with adjacent ethmoid sinusitis. Transnasal ethmoid biopsy disclosed mucormycosis. A left external ethmoidectomy, maxillectomy, and orbital exploration were performed, after which the patient was treated with daily intravenous amphotericin B for six weeks. Coexistence of retinal and nasal posterior ciliary artery occlusion due to mucormycosis may relate to their common origin from the ophthalmic artery. Treatment without exenteration was successful.

Patients with orbital mucormycosis commonly present with abrupt visual loss, orbital pain, and facial hypoaesthesia. Signs of orbital mucormycosis include chemosis, periorbital cellulitis, ophthalmoplegia, hypoaesthesia, proptosis, and ptosis. ${ }^{1+}$ Treatment is generally wide debridement, including exenteration if the orbit is involved. An unusual presentation of a patient with rhino-orbital mucromycosis and management without exenteration is presented.

\section{Case report}

A 61-year-old man developed a red eye which was treated with topical Neosporin (neomycin, gramicidin, polymyxin B) ointment. One week later the patient experienced sudden, painful total loss of vision (no light perception) in his left eye. A central retinal artery occlusion was diagnosed three days later when he presented to his ophthalmologist. Extraocular movements were limited in abduction and adduction. CT scanning disclosed a diffuse enhancement at the orbital apex and some mucosal thickening in the left ethmoid sinus. A transnasal ethmoid biopsy was performed followed by a pulse of corticosteroid (prednisone, $120 \mathrm{mg}$ daily) without

Correspondence to James C Orcutt, MD, Department of Ophthalmology RJ-10, University of Washington, Seattle WA 98195, USA. improvement. The ethmoid biopsy disclosed nonseptate hyphae.

The patient was transferred to the orbit service at the University of Washington, Seattle. Visual acuity was 20/20 in the right eye and no light perception in the left eye. The left pupil was dilated and amaurotic. Extraocular movements were limited in abduction and adduction with normal forced ductions. The conjunctiva was slightly injected, but there was no proptosis. Ophthalmoscopy showed the right eye to be normal, while the left fundus had opacification of the superficial layers of the retina with a macular cherryred spot. The veins showed stasis and the arterioles were collapsed with segmentation (Fig. 1). The retinal circulation was absent on fluorescein angiography. The normally fluorescent temporal choroid was demarcated from the unfilled nasal choroid by a well defined margin running vertically between the disc and macula (Fig. 2).

Serial axial head and orbit CT scans disclosed a left retro-orbital enhancing soft tissue mass along the posterior and medial orbit. The extraocular muscles were poorly defined in the region of the mass. The globe did not appear displaced. The optic nerve was thickened and showed enhancement of the sheath. Abnormal soft tissue density was present within the left maxillary, ethmoid, and inferior frontal sinus (Fig. 3).

The past medical history included chronic active 


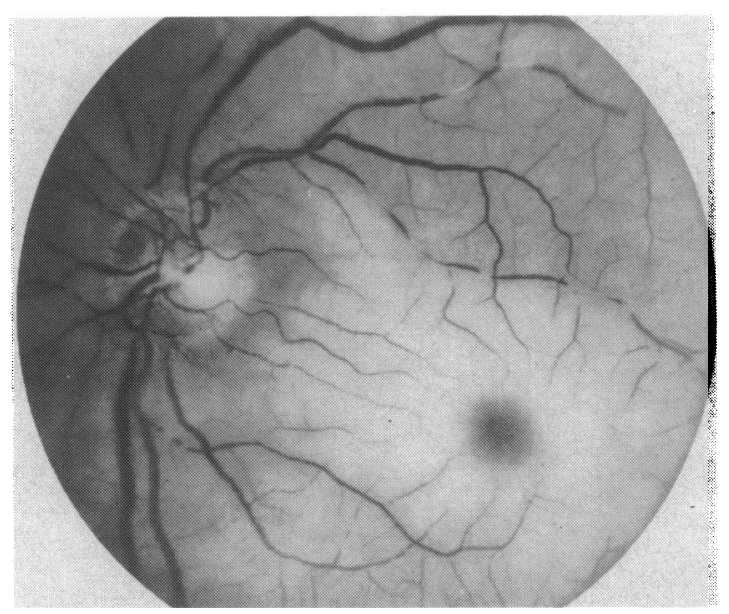

Fig. 1 Left central artery occlusion shows superficial retina oedema with a macular cherry-red spot, venous stasis, and arteriolar collapse with segmentation.

hepatitis, confirmed twice by liver biopsies, six and a half years previously. The patient had been treated continuously with corticosteroids for the past year, most recently with $20 \mathrm{mg}$ of prednisone daily. Because a metyropone suppression test disclosed suppressed adrenal function, he was continued on a

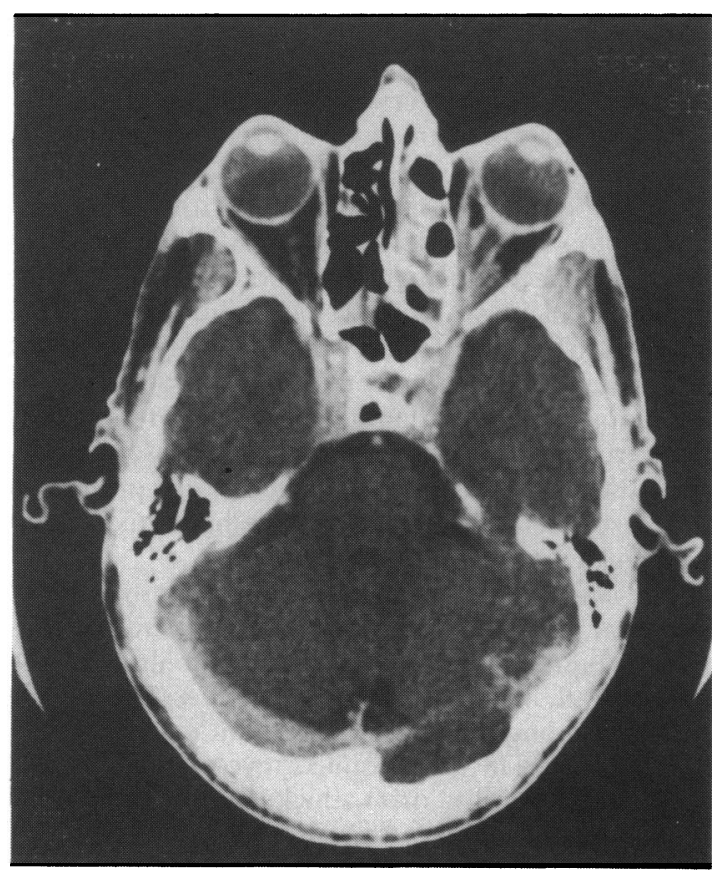

Fig. 3 CT scan shows an enhancing tissue mass along the posterior and medial aspects of the orbit, a thickened optic nerve with tramline enhancement, and soft tissue density within the left ethmoid sinus.

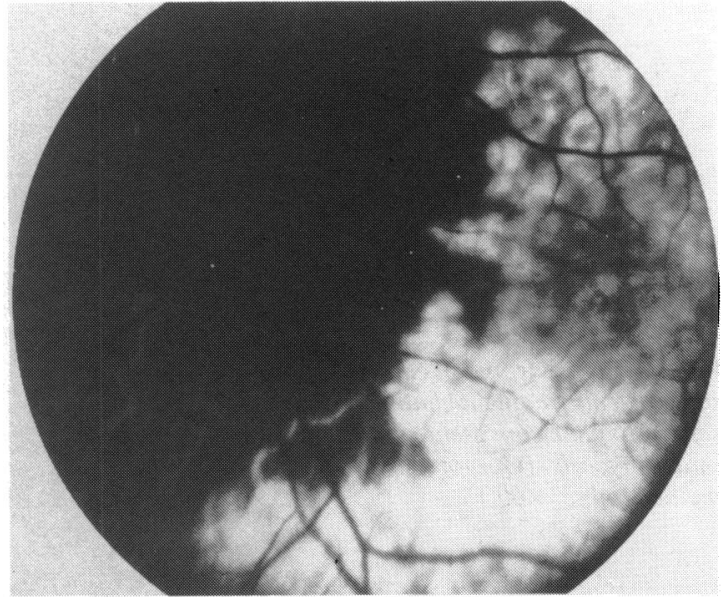

Fig. 2 Fluorescein angiography shows absence of fluorescence in the retinal vessels and nasal choroid. Normal fluorescence in present in the temporal choroid. A well defined vertical margin separates perfused and non-perfused choroid.

maintenance dosage of $5 \mathrm{mg}$ of prednisone daily. Steroid induced diabetes mellitus was being treated by diet. On admission to hospital his fasting blood glucose was $12.6 \mathrm{mmol} / \mathrm{l}(227 \mathrm{mg} / \mathrm{dl})$. The blood glucose was regulated with insulin while he was in hospital. The cerebrospinal fluid was normal, and cultures from it negative.

The patient underwent a left external ethmoidectomy and Caldwell-Luc maxillectomy. The posterior orbit was explored and found to be normal. Histopathological examination of the tissue from the ethmoid sinus disclosed fragmented non-septate hyphae compatible with mucormycosis (Fig. 4). The orbital biopsy did not show any fungal elements. Cultures of the biopsy material did not grow any organisms.

The patient completed a six-week course of intravenous amphotericin $B$. He fully recovered except for blindness in the left eye. CT scanning one year later showed the orbital mass unchanged, probably due to fibrosis.

\section{Discussion}

Mucormycosis is a known cause of central retinal and ciliary artery occlusion. Ferry and Abedi ${ }^{4}$ described an exenteration specimen of rhino-orbital-cerebral mucormycosis with thrombosed ciliary arteries next to a necrotic optic nerve. Brown et al. ${ }^{5}$ reported one of eight cases and Bullock et al. ${ }^{6}$ one of two cases with acute obstruction of the retinal and choroidal circulations in orbital mucormycosis which were not confirmed by fluorescein angiography or by histo- 
Fig. 4 Biopsy from left ethmoid sinus reveals fragmented nonseptate hyphae consistent with mucormycosis. (Gomori silver methenamine, $\times 60$.)

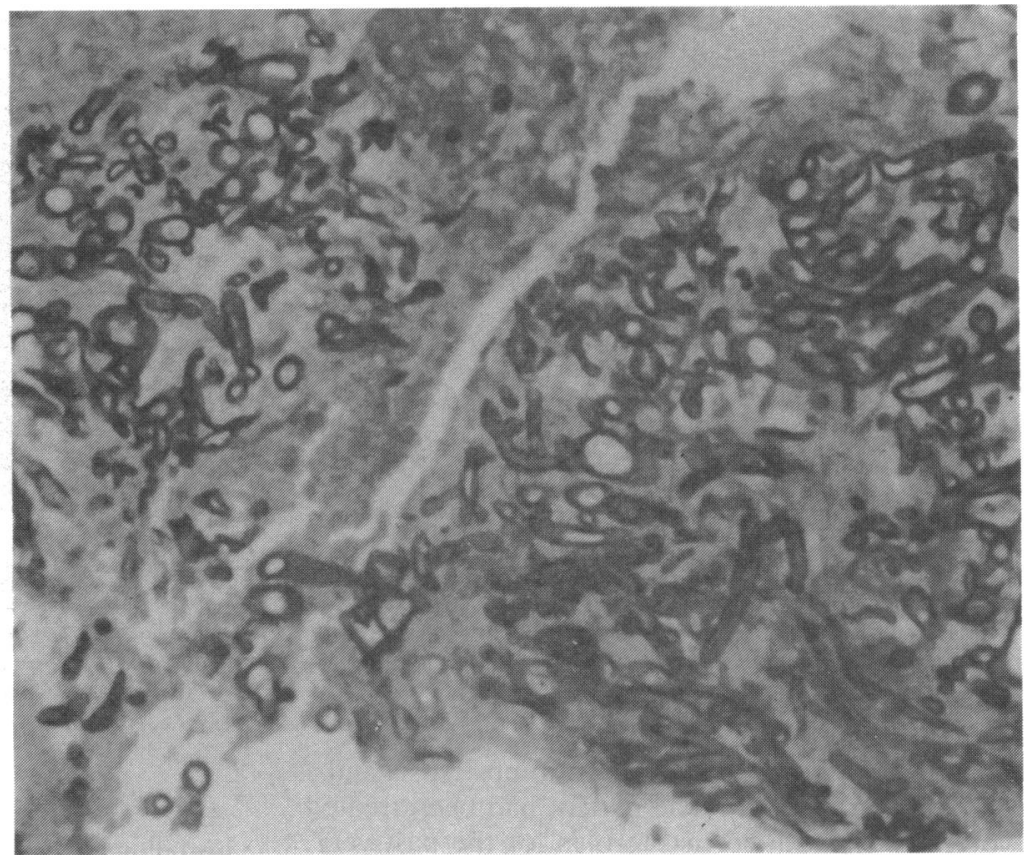

pathology. However, in our case central retinal artery and choroidal artery occlusions due to mucromycosis were confirmed by fluorescein angiography.

Mucormysosis causes a necrotising vasculitis by infiltrating the walls of blood vessels. Secondary thrombosis of the vessel lumen occurs, resulting in infarction. $^{?}$

The central retinal artery branches from the ophthalmic artery near the apex of the orbit. It runs forward and pierces the infranasal dura of the optic nerve 8 to $15 \mathrm{~mm}$ behind the globe. Two additional large arteries branch from the ophthalmic artery near the orbit apex, each subdividing into 8 to 10 ciliary branches, which pierce the sclera round the entrance of the optic nerve. ${ }^{8}$ The central retinal artery and the nasal short posterior ciliary artery arise from a common trunk of the ophthalmic artery in $40 \%$ of cases. This common trunk divides into the central retinal and nasal ciliary arteries at some distance from the opthalmic artery. In other, less common, configurations the central retinal artery may arise in common with the temporal posterior ciliary artery or with both posterior ciliary arteries. ${ }^{9}$

The short posterior ciliary arteries are arranged segmentally with each branch supplying a localised area of choroid. ${ }^{10} \mathrm{~A}$ choroidal watershed area is present where the territories of distribution of nasal and temporal posterior ciliary arteries meet between the fovea and nasal border of the optic disc. " Our patient probably had the most frequent pattern of a common branch subserving the nasal choroid and central retinal artery. Presumably the central retinal artery and nasal posterior branch were occluded by a mucormycotic necrotising vasculitis, whereas the temporal posterior artery escaped a similar fate.

Most patients with rhino-orbital mucormycosis have a predisposing systemic disease. $80 \%$ of patients have diabetes mellitus, generally with excessively raised blood glucose levels often with ketoacidosis. ${ }^{1}$ A common factor in many other predisposing states is metabolic acidosis with renal failure or gastroenteritis in infants. ${ }^{3}$ Rhino-orbital mucormycosis has also been found in patients on chemotherapy or immunosuppressive therapy. ${ }^{137}$ Mucormycosis has been reported occasionally in otherwise healthy individuals. ${ }^{212}$ Multiple risk factors were present in this case, including chronic active hepatitis treated with corticosteroids and diabetes.

The sinuses and nose are usually the initial site of infection. Orbital involvement can occur by contiguous spread through normal foramina in the medial wall. The fungi invade blood vessels, causing thrombosis and infarction of the orbital tissues. ${ }^{1}$

CT findings in orbital mucormycosis include soft tissue masses in the orbit, thickening of the medial rectus muscle, and proptosis. ${ }^{213}$ In our case CT scanning showed a poorly marginated soft tissue mass in the apex of the orbit, poor definition of the extraocular muscles in the region of the mass, and a thickened optic nerve with marginal enhancement. 
Successful treatment of rhino-orbital mucormycosis includes: (1) early diagnosis; (2) aggressive surgical debridement including exenteration, if necessary; (3) establishment of adequate sinus drainage; (4) intravenous amphotericin B; and (5) control of the predisposing systemic disease $.^{1-4,-7} 12-15$ Early diagnosis of rhino-orbital mucormycosis is crucial for a successful outcome, allowing prompt initiation of appropriate treatment. Debridement of local areas of infection and necrotic tissue, with drainage of infected paranasal sinuses, is important because local vascular thrombosis prevents systemically administered drugs from reaching the infected tissues. Exenteration is not, however, required in all cases of orbital mucomycosis. Bullock et al. ${ }^{6}$ reported two cases of rhino-orbital mucormycosis treated successfully without exenteration and with sparing of vision. Eight cases of rhino-orbital mucormycosis managed successfully without exenteration were reported by Kohn et al. ${ }^{7}$

The survival rate among patients with mucormycosis not treated with amphotericin B was $14 \%$, whereas $57 \%$ of the patients treated with amphotericin B survived. ${ }^{1}$ In our case retinal artery occlusion was noted five days before initiation of intravenous amphotericin B. Exenteration was not performed because of the normal appearance of the orbital contents and no evidence of fungal elements on biopsy. The patient had been treated with amphotericin $\mathrm{B}$ for two days preceding the biopsy, which may explain the absence of fungal elements in the orbit. He recovered but without return of vision. CT scanning one year later showed the orbital apex appearance unchanged; the persistent mass is probably due to fibrosis. The CT appearance, therefore, was not a reliable indicator of the adequacy of therapy.
This study was supported in part by an award from Research to Prevent Blindness, Incorporated.

\section{References}

1 Schwartz JN, Donnelly EH, Klintworth GK. Ocular and orbital phycomycosis. Surv Ophthalmol 1977: 22: 3-28.

2 Zak SM, Katz B. Successfully treated spheno-orbital mucormycosis in an otherwise healthy adult. Ann Ophthalmol 1985; 17: 344-8.

3 Bray WH, Giangiacomo J, Ide CH. Orbital apex syndrome. Surv Ophthalmol 1987; 32: 136-40.

4 Ferry AP, Abedi H. Diagnosis and management of rhinoorbitocerebral mucormycosis. A report of 16 personally observed cases. Ophthalmology 1983; 90: 1096-104.

5 Brown GC, Magargal LE, Sergott B. Acute obstruction of the retinal and choroidal circulations. Ophthalmology 1986; 93: 1373-82.

6 Bullock JD, Jampol LM, Fezza AJ. Two cases of orbital phycomycosis with recovery. Am J Ophthalmol 1974; 78: 811-3.

7 Kohn R, Hepler R. Management of limited rhino-orbital mucormycosis without exenteration. Ophthalmology 1985; 92: $1440-4$.

8 Reeh MJ, Wobig JL, Wirtschafter JD. Ophthalmic anatomy. A manual with some clinical applications. AAO continuing education in ophthalmology. Rochester: Custom Printing, 1981: 8691.

9 Hayreh SS. Anterior ischaemic optic neuropathy: II. Fundus on ophthalmoscopy and fluorescein angiography. Br J Ophthalmol 1974; 58: 964-80.

10 Wise GN, Dollery CT. Henkind P. The retinal circulation. Philadelphia: Harper and Row, 1971: 56-7.

11 Hayreh SS. Acute choridal ischaemia. Trans Ophthalmol Soc UK 1980; 100: 400-7.

12 Baum JL. Rhino-orbital mucormycosis occurring in an otherwise apparently healthy individual. Am J Ophthalmol 1967; 63: 355-9.

13 Greenberg MR, Lippman SM, Grinnell V, et al. Computed tomographic findings in orbital mucor. West J Med 1985; 143: $102-3$.

14 Blitzer A, Lawson W, Meyers BR. Patient survival factor in paranasal sinus mucormycosis. Laryngoscope 1980; 90: 635-48.

15 Weber PA, Makley TA, Werling K. Cerebro-rhino-orbital phycomycosis: a case report. Ann Ophthalmol 1980; 12: 459-63.

Accepted for publication 18 November 1988. 